\title{
Assessing Nurses' Work-life Balance of a Regional Teaching Hospital in Taiwan
}

\author{
Yii-Ching LEE ${ }^{1,2, a}$, Chih-Hsuan HUANG ${ }^{3,4, b}$ and Hsin-Hung WU ${ }^{5, c, *}$ \\ ${ }^{1}$ Department of Health Business Administration, Hung Kuang University, Taichung City, Taiwan \\ ${ }^{2}$ School of Health Policy and Management, Chung Shan Medical University, Taichung City, Taiwan \\ ${ }^{3}$ School of Business Administration, Hubei University of Economics, Wuhan City, China \\ ${ }^{4}$ Institute for Development of Cross-Strait Small and Medium Enterprise, Hubei University of \\ Economics, Wuhan City, China \\ ${ }^{5}$ Department of Business Administration, National Changhua University of Education, Changhua, \\ Taiwan \\ ayiiching.lee@gmail.com, btititacer@hbue.edu.cn, chhwu@cc.ncue.edu.tw \\ ${ }^{*}$ Corresponding author
}

Keywords: Burnout, Work-life balance, Safety attitudes questionnaire, Analysis of variance.

\begin{abstract}
This study examines if nurses with different demographic variables have different work-life balance by using seven questions of burnout dimension from the Chinese version of safety attitudes questionnaire in a regional teaching hospital in Taiwan with the 2015 internal data. The results show that gender (with the unequal variance assumption), supervisor/manager, reporting events in the past 12 months, job status, and experience in organization have significant influences on late work. Job status and experience in organization have significant impacts on individual or family plan change due to work factors. Besides, reporting events in the past 12 months has a significant impact on missed meals. Therefore, late work should be placed in the first priority followed by individual or family plan change due to work factors and missed meals in order to improve nurses' work-life balance.
\end{abstract}

\section{Introduction}

Mennino et al. [1] stated that the atmosphere of the workplace (culture) is an important factor to result in negative spillover in both home-to-job and job-to-home directions. The negative spillover indicates there is a work-family conflict. That is, employees might try to find a balance between their jobs and families. In fact, a person who has a negative spillover in family might not be able to concentrate on his or her job. On the other hand, if a person is stressful in his or her job, the life is to be influenced negatively.

AbuAlRub and Alhijaa [2] pointed out that nurses are the biggest workforce in the healthcare organizations and can possess enough power to move the organizational culture toward a better patient safety culture. Thus, it is of interest to observe the work-life balance from nurses' viewpoints. Nurse burnout is associated with patient healthcare quality and job dissatisfaction, and an increase in a nurse' workload could cause higher infections [3]. Moreover, Shanafelt et al. [4] showed that medical errors are highly related to the degree of burnout and mental quality of life.

The Chinese version of safety attitudes questionnaire (SAQ) developed by the Taiwan Joint Commission on Hospital Accreditation has eight dimensions including burnout dimension with seven questions that is used to assess the medical staffs' work-life balance in healthcare organizations in Taiwan annually [5,6]. The study conducted by Mennino et al. [1] showed that different demographic variables might have different impacts on spillover. Due to different demographic variables, it is of interest to assess how nurses perceive in work-life balance in terms of seven questions in burnout dimension by using independent sample $t$ test for mean differences and one-way analysis of variance (ANOVA). 


\section{Literature Review}

The Chinese version of SAQ has eight dimensions. Six of eight dimensions that consist of teamwork climate, safety climate, job satisfaction, stress recognition, perceptions of management, and working conditions with thirty questions are from Sexton et al. [7]. The seventh dimension borrowed from Maslach burnout inventory-human services survey is emotional exhaustion with nine questions to assess medical staffs' degree of fatigue [6]. The eighth dimension is burnout which assesses the medical staffs' work-life balance with seven questions: 1 . Missed meals, 2. A hasty meal, 3. All-day work without any rest, 4 . Individual or family plan change due to work factors, 5. Poor sleep, 6. Less than five-hour sleep at night, and 7. Late work [6].

Except for burnout dimension, each respondent was asked to assess each dimension by a five-point Likert scale from strongly disagree with a numerical value of one to strongly agree with a numerical value of five. In contrast, burnout dimension uses a four-point scale that measures the frequency per week, such as less than one day per week, 1-2 days per week, 3-4 days per week, and 5-7 days per week with the numerical values of $1,2,3$, and 4, respectively [6]. That is, a higher value indicates the employee is toward the work-life imbalance. In addition, there are ten demographic variables in the Chinese SAQ survey including job position [6]. In this study, job position focuses on nurses solely.

\section{Research Method}

Nurses with different demographic variables of a regional teaching hospital in Taiwan are assessed if they have different perceptions on burnout based on the internal survey results conducted in October-November 2015 from the Chinese version of SAQ. The number of effective questionnaires is 334. The frequencies and percentages in each demographic variable are depicted in Table 1.

Table 1 Distributions of demographic variables of this case hospital

\begin{tabular}{|l|l|r|r|}
\hline \multicolumn{2}{|c|}{ Demographic Variable } & Frequency & Percentage \\
\hline Gender & 1. Male & 18 & 5.4 \\
& 2. Female & 316 & 94.6 \\
\hline Age & 1. Less than 20 years old & 8 & 2.4 \\
& $2.21-30$ years old & 139 & 41.6 \\
& 3. 31-40 years old & 125 & 37.4 \\
& $4.41-50$ years old & 53 & 15.9 \\
& $5.51-60$ years old & 9 & 2.7 \\
& 6.61 years old and above & 0 & 0 \\
\hline Supervisor/Manager & 1. Yes & 28 & 8.4 \\
& 2. No & 306 & 91.6 \\
\hline Reporting events in & 1. No & 205 & 61.4 \\
the past 12 months & 2.1-5 & 113 & 33.8 \\
& 3.6-10 & 14 & 4.2 \\
& $4.11-15$ & 2 & 0.6 \\
& 5. More than 15 & 0 & 0 \\
\hline Job status & 1. Full time & 303 & 90.7 \\
& 2. Part time & 11 & 3.3 \\
& 3. Agency & 6 & 1.8 \\
& 4. Contact & 14 & 4.2 \\
\hline Experience in & 1. Less than 6 months & 54 & 16.2 \\
organization & 2.6 to 11 months & 8 & 2.4 \\
& 3. to 2 years & 53 & 15.9 \\
& 4. to 4 years & 45 & 13.5 \\
& 5.5 to 10 years & 78 & 23.4 \\
& 6. 11 to 20 years & 87 & 26.0 \\
& 7.21 years or more & 9 & 2.7 \\
\hline
\end{tabular}


Table 1(Cont.)

\begin{tabular}{|l|l|r|r|}
\hline Experience in position & 1. Less than 6 months & 61 & 18.3 \\
& 2. 6 to 11 months & 9 & 2.7 \\
& 3. 1 to 2 years & 59 & 17.7 \\
& 4. 3 to 4 years & 54 & 16.2 \\
& 5. 5 to 10 years & 90 & 26.9 \\
& 6. 11 to 20 years & 58 & 17.4 \\
& 7.21 years or more & 3 & 0.9 \\
\hline Education & 1. Junior high school and below & 0 & 0 \\
& 2. Senior high school & 1 & 0.3 \\
& 3. College/University & 322 & 96.4 \\
& 4. Graduate school and above & 11 & 3.3 \\
\hline Direct patient contact & 1. No & 10 & 3.0 \\
& 2. Rare & 20 & 6.0 \\
& 3. Very often & 304 & 91.0 \\
\hline
\end{tabular}

The scale for these seven questions in burnout ranges from one to four, where smaller values indicate a better work-life balance. In order to assess if nurses with different demographic variables perceive burnout differently from an overall viewpoints, independent sample $t$ test for mean differences with $\alpha=$ 0.05 is used to test gender and supervisor/manager. In addition, one-way ANOVA with $\alpha=0.05$ is used to evaluate the other seven demographic variables, and then Bonferroni method is chosen to perform the post hoc analysis [8].

\section{Research Results}

Independent sample $\mathrm{t}$ test is used to test if nurses perceive different burnout in terms of gender and supervisor/manager. From Table 2, males and females do not perceive burnout differently except for Item 7 (late work) with the unequal variance assumption. That is, male nurses have relatively higher frequencies in late work than female nurses. From Table 3, employees who are not in charge of supervisors/managers tend to have higher frequency in late work (Item 7) statistically.

Table 2 Mean differences on burnout for gender

\begin{tabular}{|c|c|c|c|c|}
\hline Question & Assumption & $\mathrm{t}$ & Sig. & Post Hoc \\
\hline \multirow{2}{*}{1} & Equal Variance & .859 & .391 & \\
& Unequal Variance & 1.112 & .279 & \\
\hline \multirow{2}{*}{2} & Equal Variance & .685 & .494 & \\
& Unequal Variance & .781 & .444 & \\
\hline \multirow{2}{*}{3} & Equal Variance & 1.214 & .226 & \\
& Unequal Variance & 1.300 & .209 & \\
\multirow{2}{*}{4} & Equal Variance & .732 & .465 & \\
& Unequal Variance & .933 & .362 & \\
\multirow{2}{*}{5} & Equal Variance & .943 & .346 & \\
& Unequal Variance & 1.014 & .323 & \\
\multirow{2}{*}{6} & Equal Variance & .517 & .606 & \\
& Unequal Variance & .614 & .546 & \\
\multirow{2}{*}{7} & Equal Variance & 1.828 & .069 & \multirow{2}{*}{2} \\
\hline
\end{tabular}

Table 3 Mean differences on burnout for supervisor/manager

\begin{tabular}{|c|c|c|c|c|}
\hline Question & Assumption & $\mathrm{t}$ & Sig. & Post Hoc \\
\hline \multirow{2}{*}{1} & Equal Variance & -.579 & .563 & \\
& Unequal Variance & -.541 & .593 & \\
\hline
\end{tabular}


Table 3(Cont.)

\begin{tabular}{|c|c|c|c|c|}
\hline \multirow{2}{*}{2} & Equal Variance & .278 & .781 & \\
& Unequal Variance & .348 & .730 & \\
\hline \multirow{2}{*}{3} & Equal Variance & -.877 & .381 & \\
& Unequal Variance & -1.108 & .275 & \\
\hline \multirow{2}{*}{4} & Equal Variance & -.155 & .877 & \\
& Unequal Variance & -.155 & .878 & \\
\hline \multirow{2}{*}{5} & Equal Variance & -.044 & .965 & \\
& Unequal Variance & -.046 & .964 & \\
\hline \multirow{2}{*}{6} & Equal Variance & .303 & .762 & \\
& Unequal Variance & .322 & .749 & $2>1$ \\
\multirow{2}{*}{7} & Equal Variance & -3.964 & .000 & $2>1$ \\
\hline
\end{tabular}

Age does not show any significant impacts on these seven questions depicted in Table 4. Employees who report different events in the past 12 months have significant perceptions on Items 1, 2, and 7 as shown in Table 5. The post hoc analysis shows that employees who report 6-10 events have significantly lower frequencies than employees who report zero events in missed meals and late work. Table 6 summarizes how job status influences burnout. The results show that contract-based employees have significantly higher frequencies than part-time employees and full-time employees in Items 4 (individual or family plan change due to work factors) and 7 (late work), respectively.

Table 4 ANOVA results on burnout for age

\begin{tabular}{|c|c|c|c|}
\hline Item & F & Sig. & Bonferroni \\
\hline 1 & .643 & .632 & \\
\hline 2 & 1.037 & .388 & \\
\hline 3 & .498 & .737 & \\
\hline 4 & 1.835 & .122 & \\
\hline 5 & 1.020 & .397 & \\
\hline 6 & 1.450 & .217 & \\
\hline 7 & .747 & .560 & \\
\hline
\end{tabular}

Table 5 ANOVA results on burnout for reporting events in the past 12 months

\begin{tabular}{|c|c|c|c|}
\hline Item & F & Sig. & Bonferroni \\
\hline 1 & 5.718 & .001 & $1>3$ \\
\hline 2 & 3.718 & .012 & \\
\hline 3 & 1.149 & .329 & \\
\hline 4 & 2.443 & .064 & \\
\hline 5 & 1.553 & .201 & \\
\hline 6 & 1.337 & .262 & \\
\hline 7 & 3.453 & .017 & $1>3$ \\
\hline
\end{tabular}

Table 6 ANOVA results on burnout for job status

\begin{tabular}{|c|c|c|c|}
\hline Item & F & Sig. & Bonferroni \\
\hline 1 & 1.607 & .188 & \\
\hline 2 & 2.357 & .072 & \\
\hline 3 & 2.172 & .091 & \\
\hline 4 & 2.994 & .031 & $4>2$ \\
\hline 5 & 1.596 & .190 & \\
\hline 6 & 1.222 & .302 & \\
\hline 7 & 2.751 & .043 & $4>1$ \\
\hline
\end{tabular}


Employees with different experiences in organization have different frequencies in Items 2, 4, and 7 illustrated in Table 7 statistically. In addition, the post hoc analysis shows that employees with 5 to 10 years in organization perceive lower frequencies statistically than those with less than 6 months in organization and those 3 to 4 years in organization in Items 4 and 7, respectively. From Table 8 , employees with different experiences in position have different perceptions in Items 4 and 7 . However, the post hoc analysis does not show any difference among different experiences in position. Table 9 shows that employees with different educations do not perceive burnout differently. Finally, in Table 10, employees who have direct patient contact perceive differently in Item 2 (a hasty meal), but the Bonferroni method does not show any differences among different levels of direct patient contacts.

Table 7 ANOVA results on burnout for experience in organization

\begin{tabular}{|c|c|c|c|}
\hline Item & F & Sig. & Bonferroni \\
\hline 1 & 1.782 & .102 & \\
\hline 2 & 2.520 & .021 & \\
\hline 3 & 1.972 & .069 & \\
\hline 4 & 2.550 & .020 & $1>5$ \\
\hline 5 & 1.321 & .247 & \\
\hline 6 & 1.272 & .270 & $3>5$ \\
\hline 7 & 3.041 & .007 & \\
\hline
\end{tabular}

Table 8 ANOVA results on burnout for experience in position

\begin{tabular}{|c|c|c|c|}
\hline Item & F & Sig. & Bonferroni \\
\hline 1 & 1.251 & .280 & \\
\hline 2 & 1.383 & .221 & \\
\hline 3 & 1.728 & .114 & \\
\hline 4 & 2.276 & .036 & \\
\hline 5 & .895 & .499 & \\
\hline 6 & .691 & .657 & \\
\hline 7 & 2.559 & .020 & \\
\hline
\end{tabular}

Table 9 ANOVA results on burnout for education

\begin{tabular}{|c|c|c|c|}
\hline Item & F & Sig. & Bonferroni \\
\hline 1 & .129 & .879 & \\
\hline 2 & .588 & .556 & \\
\hline 3 & .265 & .767 & \\
\hline 4 & .063 & .939 & \\
\hline 5 & .619 & .539 & \\
\hline 6 & 1.271 & .282 & \\
\hline 7 & .079 & .924 & \\
\hline
\end{tabular}

Table 10 ANOVA results on burnout for direct patient contact

\begin{tabular}{|c|c|c|c|}
\hline Item & F & Sig. & Bonferroni \\
\hline 1 & 1.475 & .230 & \\
\hline 2 & 3.826 & .023 & \\
\hline 3 & 2.593 & .076 & \\
\hline 4 & 1.700 & .184 & \\
\hline 5 & 2.327 & .099 & \\
\hline 6 & 1.900 & .151 & \\
\hline 7 & .253 & .777 & \\
\hline
\end{tabular}




\section{Conclusions}

The purpose of this study is to examine if nurses have different work-life balance by using these seven questions of burnout dimension from the internal surveyed data in 2015 of a regional teaching hospital based on the Chinese SAQ survey. The results show that gender (with the unequal variance assumption), supervisor/manager, reporting events in the past 12 months, job status, and experience in organization have significant impacts on late work. Besides, job status and experience in organization have significant influences on Item 4 (individual or family plan change due to work factors). Further, reporting events in the past 12 months has an impact on missed meals significantly. Item 7 (late work) should be placed in the highest priority in order to improve nurses' work-life balance followed by Item 4 and Item 1 (missed meals).

\section{Institutional Review Board Approval}

The clinical trial approval certificate (ethic statement) was approved by Cheng Ching General Hospital in Taichung City, Taiwan with protocol number of HP150029.

\section{Acknowledgement}

This study with the grant number of MOST 106-2221-E-018-022 was supported in part by Ministry of Science and Technology in Taiwan.

\section{References}

[1] S.F. Mennino, B.A. Rubin, A. Brayfield, Home-to-job and job-to-home spillover: The impact of company policies and workplace culture, Sociol. Quart. 46 (2005) 107-135.

[2] R.F. AbuAlRub, E.H.A. Alhijaa, The impact of educational interventions on enhancing perceptions of patient safety culture among Jordanian senior nurses, Nurs. Forum 49(2) (2014) 139-150.

[3] J.P. Cimiotti, L.H. Aiken, D.M. Sloane, E.S. Wu, Nurse staffing, burnout, and health care Associated infection, Am. J. Infect Control 40 (2012) 486-490.

[4] T.D. Shanafelt, C.M. Balch, G. Bechamps, T. Russell, L. Dyrbye, D. Satele, P. Collicott, P.J. Novotny, J. Sloan, J. Freischlag, Burnout and medical errors among American surgeons, Ann. Surg. 251(6) (2010) 995-1000.

[5] Y.C. Lee, S.J. Weng, C.H. Huang, W.L. Hsieh, H.H. Wu, Analyzing emotional exhaustion from viewpoints of physicians and nurses - A case of a regional teaching hospital, TEM J. 5(2) (2016) 231-235.

[6] Y.C. Lee, C.Y. Wang, S.J. Weng, C.C. Huang, W.L. Hsieh, H.H. Assessing patient safety culture from nurses' viewpoints of a teaching hospital in Taiwan, 5th International Conference on Application of Information and Communication Technology and Statistics in Economy and Education, 2015, 37-44.

[7] J.B. Sexton, R.L. Helmreich, T.B. Neilands, K. Rowan, K. Vella, J. Boyden, P.R. Roberts, E.J. Thomas, The safety attitudes questionnaire: Psychometric properties, benchmarking data, and emerging research, BMC Health Serv. Res. 6 (2006) 44.

[8] M.L. McHugh, Multiple comparison analysis testing in ANOVA, Biochem. Medica 21(3) (2011) 203-209. 